and relative emphasis of the topics. Another author might have devoted much more space to the "excluded volume problem" and to the various attempts that have been made to solve it. It is now known that the equilibrium configurations of a freely jointed chain are affected, qualitatively as well as quantitatively, if the interactions between distant segments are properly allowed for, but the mathematics of this is still rudimentary.

Flory has in fact concentrated on the easier problem of relating polymer properties to the properties of the "hinges" between successive "segments" of the molecule. There are, in fact, very striking analogies between the theory of a polymer chain and that of an imperfect gas, Flory's "theta-point" of a polymer solution being quite analogous with the Boyle point of an imperfect gas. In each case the effects of intermolecular attraction and repulsion balance out to a first approximation.

The first three chapters describe the general theory; chapter four introduces some of the "moments of the chain", for example, mean square length, mean square radius of gyration; the next three chapters are devoted to the various types of chain, symmetric, "vinyl" and polypeptide respectively; chapter eight summarizes various theoretical results; while chapter nine shows how various moment properties can be related to optical experiments and radiation scattering.

A particularly fascinating problem at the present time is to predict the actual configuration of a protein molecule given its amino-acid sequence. Chapter seven shows that some progress in this problem, which may soon become the central one of molecular biology, has already been made. It was a little surprising to find no references to equally important problems, such as radiation damage, the effect of cross-linking, and polymer degradation in general, but one cannot put the whole of a vast science into one book.

The bibliographies at the end of each chapter, the glossary of symbols, the author and subject indexes and the appendices on special topics have been prepared with great care.

The book is thoroughly recommended to all serious workers in this field. Students at honours level would also benefit but might find some of the going very tough indecd.

H. N. V. TEMPERLey

\section{NUCLEOPHILIC SUBSTITUTIONS}

\section{Aromatic Nucleophilic Substitution}

By J. Miller. (Reaction Mechanisms in Organic Chemistry, Monograph 8.) Pp. xi+408. (Elsevier: Amsterdam, London and New York, 1968.) $155 s$.

Alтнолgн there are several reviews of aromatic nucleophilic substitution now available, this latest volume in Elsevier's "Reaction Mechanisms" series is the first book devoted exclusively to the subject. It is by far the most comprchensive and detailed of these surveys, and it is thus a welcome addition to the chemical literature. It is the more welcome because Professor Miller writes with all the authority of one who, with his research collaborators, has made many important contributions to this area of chemistry.

A short introductory chapter is followed by discussion of the three principal mechanisms of substitution. $S_{N} 1$ and elimination-addition (benzyne) mechanisms are discussed briefly (32 pages), and the addition-elimination $\left(\mathrm{S}_{\mathrm{N}} 2\right)$ process is then discussed, in detail and at length (173 pages), in terms of (i) substituent effects, (ii) variation of leaving groups, and (iii) the nucleophilic reagent. Succeeding chapters deal with substitution in heteroaromatic and certain other non-benzenoid systems, and with solvent, salt and counter ion effects, catalysis, and steric effects. The concluding section describes some $S_{N}$ and other reactions which, although formally nucleophilic substitutions, do not belong to any of the previously described typcs.

Inevitably, the chapters on $\mathrm{S}_{\mathrm{N}} 2$ mechanisms, which contain a wealth of quantitative data (46 tables), make less easy reading than those of a more qualitative nature, but this situation is not improved by the layout of the tables: those which are long and complicated (for example, $34 \mathrm{~A}$ and $34 \mathrm{~B}$ ) would be much clearer if subheadings were interspersed with the data rather than collected at the top.

It is surprising to find naphthalene derivatives discussed under "non-benzenoid systems", although this presumably facilitates comparison with their aza-analogues. It is equally surprising, and disappointing in view of the current interest in aryne chemistry, that this section of the book is so small, and also that Hoffmann's comprehensive monograph on this topic ${ }^{1}$ receives no mention. And the assertion (page 92) that " $2-\mathrm{Br}-\mathrm{I}-\mathrm{NO}_{2}$-naphthalene is considerably more reactive than $2 \cdot \mathrm{Br}-3-\mathrm{NO}_{2}$-naphthalene ... because the transition state for the former retains one ring benzenoid whereas that for the latter does not" gives an over-simplified picture of the situation.

These, however, are minor faults. As a whole, the book is of immense value and is warmly recommended. It should quickly become the standard reference work for researchers in this field. The standard of production is predictably high; so, alas, is the price.

\section{M. SMith}

${ }^{1}$ Hoffmann, R. W., Dehydrobenzene and Cycloalkynes (Academic Press, 1967).

\section{SHORTENED CHEMISTRY}

\section{A Shorter Sidgwick's Organic Chemistry of Nitrogen} Revised and rewritten by Ian T. Millar and H. D. Springall. Pp. xi +582 . (Clarendon Press: Oxford; Oxford University Press: London, May 1969.) $40 s$.

The third edition of Sidgwick's classical Organic Chemistry of Nitrogen appeared in 1966 as a completely revised and rewritten book. Its authors, Professors Millar and Springall, spent many years on what turned out to be a mammoth task. It was immediately apparent, however, that its size and price would preclude its presence from the bookshelves of honours students, who, it was hoped, would comprise a large section of its readership. To rectify this shortcoming, a shortened version of this third edition has been prepared and with commendable speed.

During the lifetimes of the first and second editions the book fulfilled a dual role. It served as an invaluable source for honours students in departments where natural product chemistry was taught in extenso, and it maintained this function at the postgraduate level. The third edition failed to score on both points for two reasons. First, the immense growth of the subject over the past thirty years made for a much larger book, and second, the current availability of specialized texts on various parts of the subject. Pre-war these texts were few and far between; now over half the chapters in the book are covered by monographs.

If the original type of book were to survive, it was clearly to the honours student that it should be aimed. Here the authors score well in this shortened third edition. In all there are seventeen chapters, but it seems pointless to list them all. Suffice it to say that they range in subject from the classical amino- and nitro-compounds, the heterocyclic systems such as pyrrole and pyridine through to the more recent protein and nucleic acid chemistry. Preparations and properties of compounds, as in earlier editions, form the basis of the book. Where possible the authors have said something about the mechanisms of the reactions involved, though they have 